\title{
Research on target detection methods in remote sensing image
}

\author{
Zhuo Chen ${ }^{1, a}$, Xiangxu Meng ${ }^{1, b}$, Xi Wang $^{1, c}$ \\ ${ }^{1}$ Department of Information Engineering, Academy of Armored Forces Engineering, Beijing, China \\ aellen_nehc@yahoo.com, ${ }^{\mathrm{b}}$ mengxx5100@163.com, ${ }^{\mathrm{C}}$ lycis@126.com
}

Keywords: Remote sensing, target detection.

Abstract. This paper mainly discusses the imaging features of remote sensing images, as well as the core target detection technology of image application, and uses several traditional image detection algorithms to experiment. The experiment results show, for unspecific targets, the edge detection algorithm based on wavelet transform has better identification and robustness than other traditional image detection algorithms. For specific targets, such as roads, bridges and airport runways, the Hough algorithm has high detection accuracy, compared with the traditional image detection algorithm.

\section{Introduction}

Remote sensing technology is emerging in the 20th century 60's developed a comprehensive scientific research subject, which is closely linked with space, geography, optoelectronics, computer science technology. It is a very active and popular current subject in the field of information. The main feature of the remote sensing technology is: no direct contact with the related target can get a lot of useful information, and to test the acquisition of data, classification and recognition; has strong macroscopic, complete information recording ability, short work cycle, and the ability to rapidly accumulating data information. This all fully highlighted the advantages of remote sensing technology [1].

With the continuous development of remote sensing technology, especially the data obtained from the space and time resolution, the corresponding data volume also increased dramatically, the traditional artificial interpretation has been difficult to accomplish such a huge data processing, so need through a variety of image processing technique for automatic accurate interpretation[2]-[5].

\section{Remote sensing image imaging features}

Classic image feature has color, shape, size, shadow and texture, the related features such as position and activity trace and so on, is to identify the various objects and factors. Remote sensing image classification methods are many. According to the imaging way, can be divided into: photographic images, scans, and radar images. According to the height of the imaging platform, can be divided into: satellite image, aerial image, and ground image. As a result of the difference of remote sensing imaging platform and technology system, imaging features and the value of feature will be some changes. Following are the imaging features of aerial photographic images, satellite scan images, and SAR images.

Aerial photographic image imaging features: 1) color feature, such as black and white panchromatic, true color, false color; 2) size; 3) shape; 4) shadow; 5) texture; 6) related position, for example, shipyard to set up by the river, lake and sea, or road and ditch, river bridge intersection generally exist; 7) activity trace, for example, according to the ship movement aroused waves can determine the direction of travel; or according to the surface traces of base area can determine the size of the past army. 


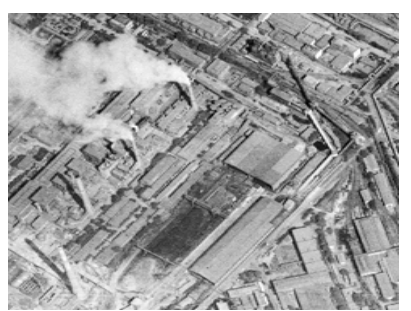

Fig.1 Judge the chimney position by smoke

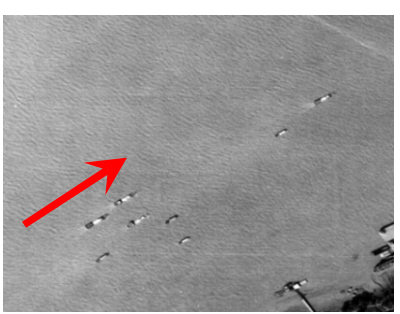

Fig.2 Judge the driving direction of ship by spray

Satellite scan image imaging features: since the satellite orbit is very high and the scanning angle is small, the position of the target on the image changes little relative to the position of the lens, so that only the top shape of the target is found on the vertical down view. For plane targets (such as road, squares, rivers, etc.), there is no too big obstacle. For targets higher than the ground, the shadow of the target not only reflects the side shape of the target, but also reflects the height of the target, which is particularly useful for target detection. As shown in the following figures: tall buildings, people's heroes' monument, flag and flagpole.
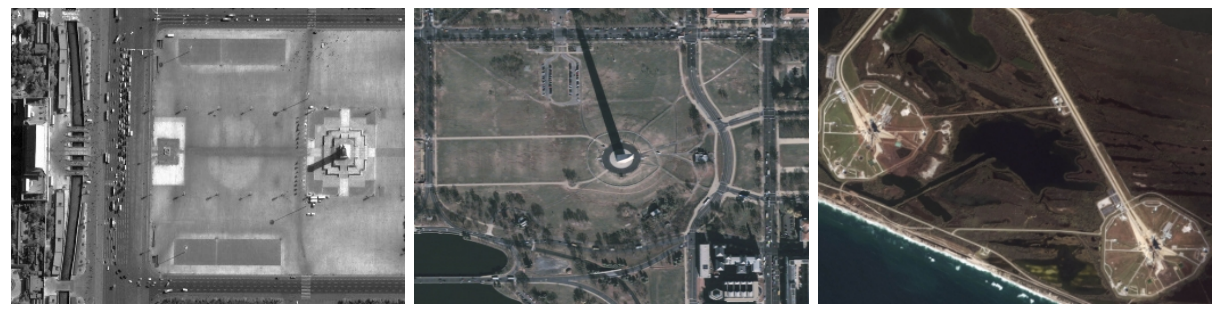

Fig.3 Satellite scan images

SAR image imaging features: because the imaging mechanism of SAR is very unique, and using a longer wavelength of microwave imaging $(1 \mathrm{~mm}-1 \mathrm{~m})$, so the SAR images also look different. 1) Most of the plane targets, such as airport runway, cottage, etc., will appear mirror reflection and have no echo. Although they are gray in full color image, but they are black in SAR image; 2) Projecting out of the ground targets, the face side part will produce strong echo in SAR since they form the corner reflector, so they are bright white on the SAR image, meanwhile the top and the back side part are black on the SAR image; 3) High dielectric constant targets, such as metal, SAR is very sensitive to them, and they are bright white on SAR image, so in SAR image it is very easy to find vehicles, warships, aircraft, etc. 4) The higher water content in the target, the more lighter in image tone, but the water surface is black in SAR image; 5) Target's shadow direction is consistent with radar's side view direction. With radar's side view angle increasing, target's shadow length is also increasing. Target's SAR image tone has nothing to do with the intensity of the sunlight.
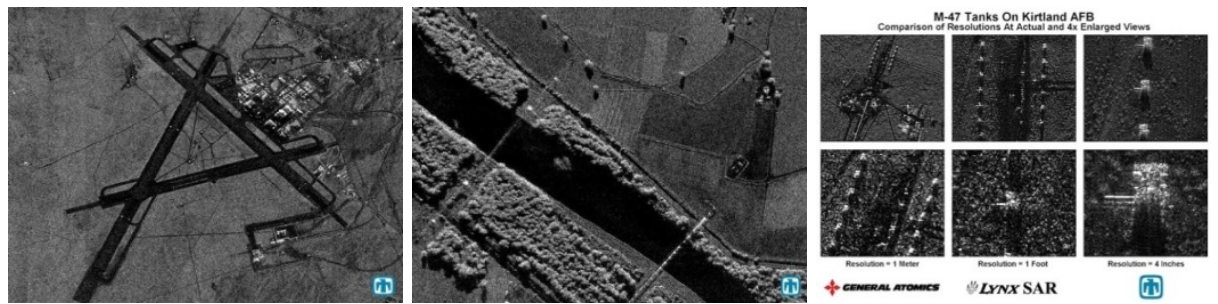

Fig.4 SAR images

\section{Remote Sensing Image Detection Methods and Effects For unspecific targets}

Extracting useful information from remote sensing image is currently the core technology in remote sensing image technology using. It is called target detection in remote sensing image processing and target recognition system. Usually, target detection is achieved by matching the feature of the extracted image with the feature of the target. The extracted features has edge feature, texture feature, regional feature and direction feature etc. On the other hand, ground targets observed by remote sensing 
platform include the following categories: 1) city target; 2) airport main building facilities; 3) ship target; 4) seaport and seaport base; 5) mountain and forest landscape. In military applications, those important targets of the ground battlefield that are be monitored and detection, are including but not limited to: roads, airports, military bases, tank cluster, convoys, missile launcher, etc. Since military battlefield requires higher real-time and effectiveness of targets detection, so the algorithm which has fast operation, high detection rate, with good robust, has become the current research hotspot [6].

At present, the edge detection method is often used in general target detection, such as road, river and coastline. The traditional edge detection algorithms are Canny, Sobel, Robert, Laplacian, etc. The following is the detection effect by using these traditional image processing edge detection operators on the unspecific targets.

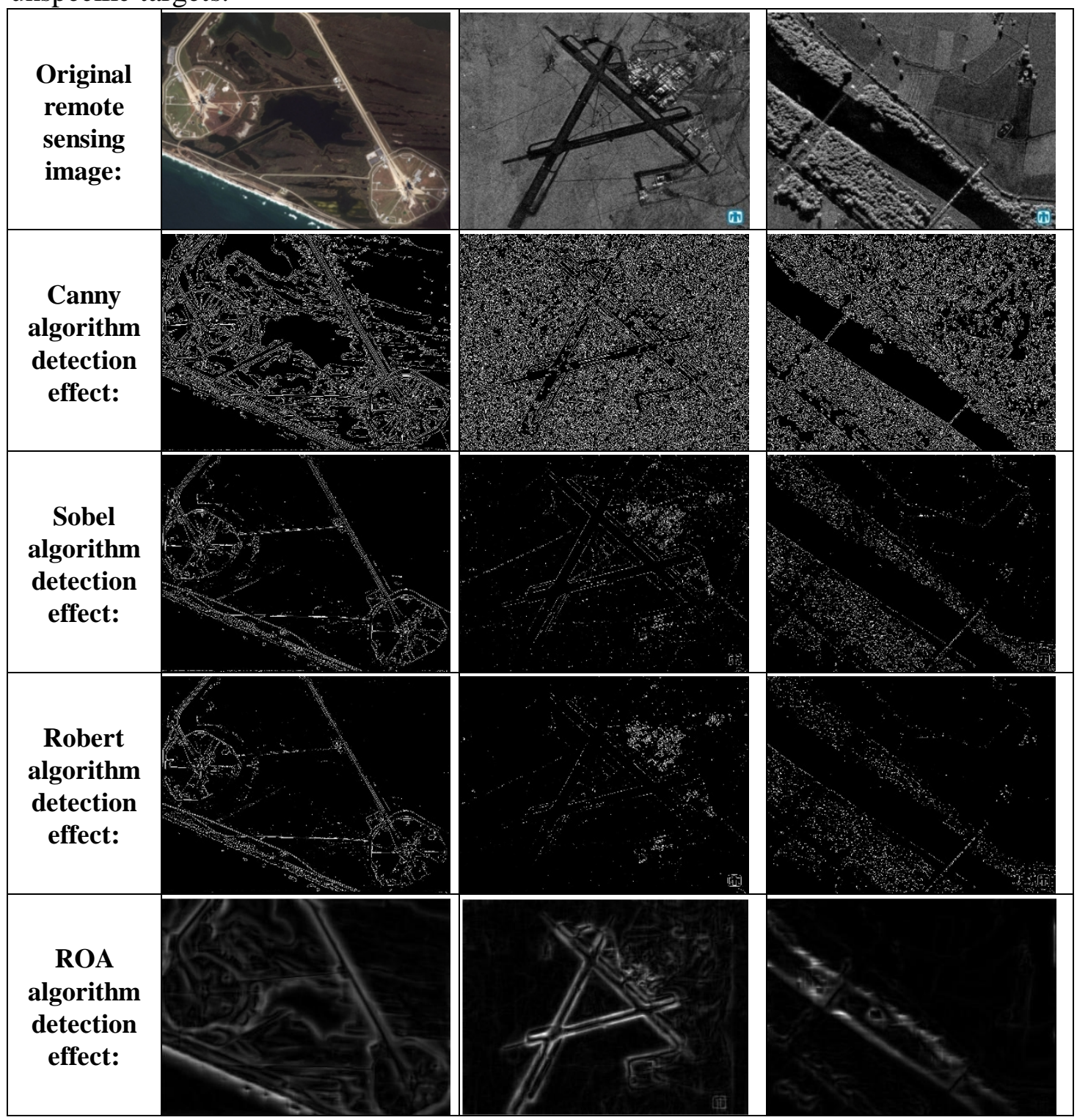

Fig.5 Traditional edge detection on Remote Sensing Image for unspecific targets

The common feature of these algorithms is to perform a certain weighting operation on a small neighborhood of each pixel of the image in the spatial domain, as a result of the edge intensity. Canny algorithm, essentially, is a first order differential operator with smoothing function, which has good signal-to-noise ratio and detection accuracy. Sobel algorithm can not only detect the edge points, but also to further suppress the noise response. However, it will be found the detected edge has a little widening, which affects the shape of the target. It is not hard to see the problem in other algorithm experiments, that the results are generally sensitive to noise. It is mainly because the use of the algorithm template is small, that cannot effectively eliminate the impact of image noise. In another 
word, the noise response is normally bigger than the edge response of the image, so the detected edge result is fuzzy.

\section{Improved Detection method}

To solve this problem, the author tests the above targets by using an improved edge detection algorithm based on wavelet transform. The algorithm based on double threshold method of the wavelet transform is a kind of automatic threshold setting, using the local property of wavelet transform, to achieve the precise positioning of the image noise reduction and image edge. The following figures are the detection effect.
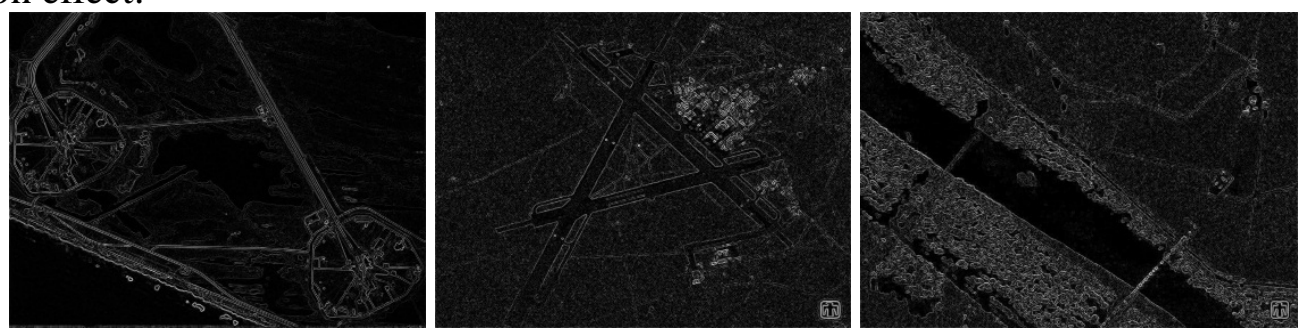

Fig.6 Double threshold wavelet transform algorithm on Remote Sensing Image for unspecific targets

Obviously, the improved edge detection algorithm based on wavelet transform is very successful for core target in the main large target area, compared with the traditional edge detection algorithms.Otherwise, there is a class of targets such as road targets or airport base runway targets, and they have a certain particularity, that is, a certain linear graphics linear retention process. It is found that for this type of target, Sobel and Canny has better detection effect than other common algorithms, although there are some small errors, such as figure 5. Thus, the author tries to test the Hough transform algorithm for such specific targets,
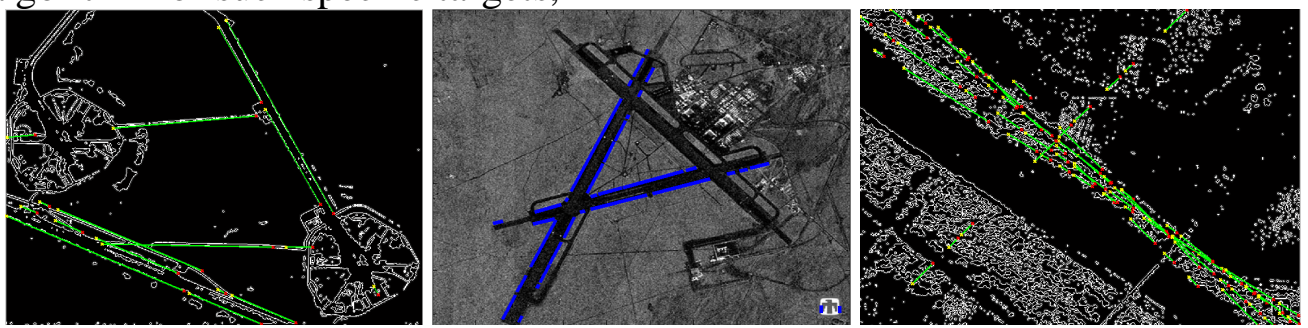

Fig.7 Double threshold wavelet transform algorithm on Remote Sensing Image for unspecific targets

The traditional Hough transform edge detection algorithm can achieve very significant effect on remote sensing images with typical road characteristics, and the effect graphs are simple and intuitive, but the algorithm is also very sensitive to image noise. So the author further improved. Firstly, the image is preprocessed by image enhancement technique, and the edge of the image is highlighted. Secondly, the multi-scale morphological image is processed, and the gray scale images are expanded and corroded by using various structural elements. The results of each operation are processed synthetically. The edge of the image is obtained. Finally, the edge detection based on Hough transform is carried out to detect the possible path.

As the algorithm increases the multi-scale morphological edge detection of this technical link, and in the image processing mainly to the image of the morphological characteristics of the study object, so the edge of the image in this aspect than the differential operation based on the edge of the extraction algorithm It is mainly because the morphological method is not particularly sensitive to noise, so the extracted edges are smoother, and the edge of the image extracted by morphological method is continuous, the processing speed is fast and the algorithm idea is clear.

The experiment results show that the number of false lines extracted by this algorithm can be reduced effectively, and the typical road feature information can be extracted effectively. It has the advantages of less directional dependence on the edge and more precise extraction boundary 


\section{Conclusions}

Through the above experiments, it is found that the edge detection algorithm based on Wavelet algorithm has better identification and robustness for unspecific target compared with the other classical image detection algorithms. On the other hand, it is found that the Hough algorithm has higher detection accuracy compared with the classical image detection algorithm for a specific target, such as roads, bridges, airport runways.

\section{References}

[1] Xie Shousheng. Microwave Remote Sensing Technology and Application [M]. Beijing: www.sciencep.com, 1987.

[2] C.J.Oliver,S.Quegan. Understanding Synthetic Aperture Radar Images[C]. Artech House Inc.685 Canton Street, Norwood, MA 02062, 1998.

[3] Song Jianguo, Zheng Shui'an, Ai Lihai. Understanding and Applications of Synthetic Aperture Radar Images. Beijing: www.sciencep.com, 2008.

[4] Jiao LiCheng. Intelligent SAR Image Processing and Interpretation[M]. Beijing: www.sciencep.com, 2008.6.

[5] Henri Maitre. Processing of Synthetic Aperture Radar Images[M]. Beijing: www.sciencep.com, 2005: 67 76.

[6] Cui Peng. Detection of vehicle targets in SAR images. Nanjing: Nanjing University of Aeronautics and Astronautics,2010:32 34. 